Musées, Patrimoine et Culture scientifiques et techniques

$126 \mid 2009$

novembre - décembre 2009

\title{
Genèse de l'Observatoire du Patrimoine et de la Culture Scientifiques et Techniques
}

Genesis of the Heritage and Scientific and Technical Culture Observatory

Florence Belaën, Fabien Lacaille et Jean-Louis Lacroix

(2) OpenEdition

Journals

Édition électronique

URL : http://journals.openedition.org/ocim/220

DOI : 10.4000/ocim.220

ISSN : 2108-646X

Éditeur

OCIM

Édition imprimée

Date de publication : 1 novembre 2009

Pagination : 15-23

ISSN : 0994-1908

Référence électronique

Florence Belaën, Fabien Lacaille et Jean-Louis Lacroix, « Genèse de l'Observatoire du Patrimoine et de la Culture Scientifiques et Techniques », La Lettre de I'OCIM [En ligne], 126 | 2009, mis en ligne le 01 novembre 2011, consulté le 19 avril 2019. URL : http://journals.openedition.org/ocim/220 ; DOI : $10.4000 /$ ocim. 220 


\section{Genèse de l'Observatoire du Patrimoine et de la Culture Scientifiques et Techniques}

Florence Belaën *

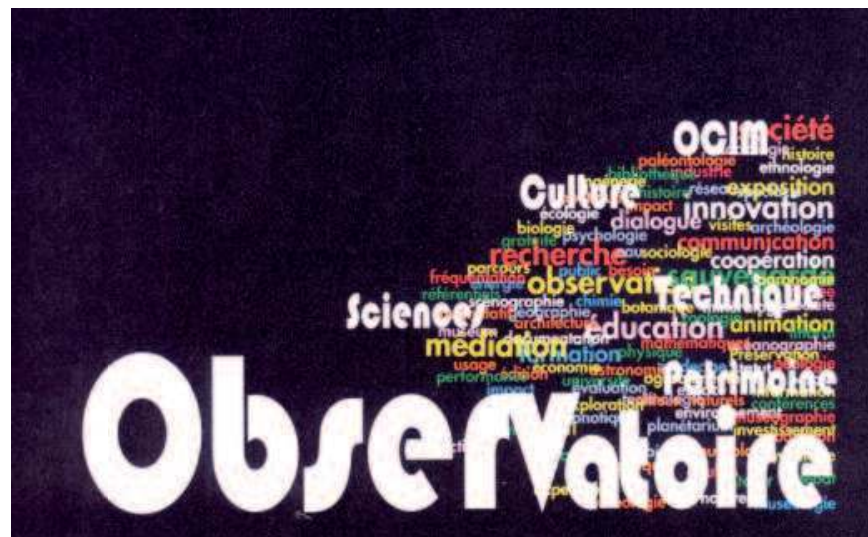

\section{Obrenver, questionner, aceompagnero..} un obserratoire pour la culture reientifique et technique

Rencontres a Dijon

les 26 et 27 novembre 2009

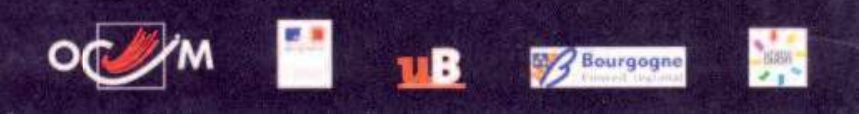

* Florence Belaën est chef de projet Observatoire du Patrimoine et de la Culture Scientifiques et Techniques florence.belaen@u-bourgogne.fr
L'OCIM a reçu une nouvelle mission : celle d'un Observatoire du Patrimoine et de la Culture Scientifiques et Techniques. Partant d'une interrogation sur la définition d'un observatoire, cet article présente les premières réflexions et pistes de travail qui animent la gestation de ce nouveau dispositif, au service des acteurs de ce champ spécifique.

Observatoires :

de la diversité aux éléments structurants

Observatoire du stress, observatoire des inégalités salariales, observatoire de l'environnement, observatoire de la vie étudiante, observatoire de la musique, observatoire des territoires, observatoire de la chanson française, observatoire des habitudes alimentaires, observatoire de la santé, observatoire des conjonctures économiques, observatoire du tourisme, observatoire de l'école rurale, observatoire des pôles de compétitivité (1)... La liste des observatoires est longue et ne manque ni d'originalité, ni de diversité. Que signifie cette mode de dispositifs « méta », largement amplifiée depuis une vingtaine d'années ? Laisserait-elle entrevoir que le besoin d'observation est symptomatique de nos sociétés contemporaines marquées par une profusion d'information et une complexité croissante?

Comme sa référence au dispositif astronomique le rappelle, la fonction principale de tout observatoire est de "considérer quelque chose avec attention pour mieux le connaître ». Mais à y regarder de plus près, 
on s'aperçoit que derrière ce terme générique - observatoire - se cache en réalité une grande variété d'outils avec des missions et des moyens hétérogènes, allant d'une « simple » mise en visibilité d'actions et d'acteurs à une organisation plus technique répertoriant finement ces mêmes éléments en vue de produire des outils stratégiques d'aide à la décision. Et si leurs missions se déclinent, leurs statuts et leurs modes de financement renvoient également à toute la palette des possibles de l'administration française et des modalités de gestion publique et privée : observatoires nationaux intégrés dans un ministère ${ }^{(2)}$, observatoires régionaux planifiés dans les contrats de Plan État-Région (3), service d'une université (4), Groupement d'Intérêt Public (5), service d'une fédération d'acteurs ${ }^{(6)}$, fonction d'une agence de développement culturel ${ }^{(7)}$, association indépendante ${ }^{(8)}$, mission d'un service d'une institution (9)... Les objets d'observation peuvent également être de nature différente, principalement deux : certains sont généralistes et d'autres centrés sur un thème. Des observatoires peuvent être axés sur la musique, le tourisme, alors que d'autres se construisent à partir d'une problématique spécifique : les habitudes alimentaires, les inégalités homme/femme. À l'intérieur de ces catégories se profilent également d'autres sous-catégories au niveau des missions des observatoires. Pour prendre l'exemple de la culture, un observatoire peut produire des analyses sectorielles (la diffusion de la danse) et intersectorielle (atlas des équipements culturels), des approches transversales des activités (les financements publics de la culture, l'emploi culturel, les formations...).

Mais dans cette famille marquée par une grande hétérogénéité de ses membres, des éléments structurants se retrouvent, notamment dans les dispositifs qui assurent au-delà d'un affichage, une réelle mission d'observation. Un des premiers dénominateurs communs à ces dispositifs est que la production de ces connaissances, quelles qu'elles soient, est toujours articulée avec une approche territoriale, apportant ainsi des éléments de contextualisation des résultats. En effet la question du territoire y est centrale en vue d'y proposer une approche comparative notamment. Ce qui explique en partie que nombreux sont les observatoires qui sont nés après une période charnière dans les politiques publiques : la décentralisation. Le transfert du pouvoir régalien aux collectivités locales a entraîné un effet de mosaïque où le schéma d'ensemble a perdu en visibilité et par conséquent, une politique commune difficilement applicable. Ainsi la décentralisation a-t-elle été un catalyseur pour l'émergence de différents observatoires, notamment des observatoires régionaux qui démontrent l'intérêt des Conseils régionaux à disposer de leurs propres outils d'analyse.

La temporalité est également essentielle pour la pratique de l'observation car la durée permet non seulement de capitaliser les informations mais de les mettre en perspective et d'en saisir l'évolution.

En plus de leur ancrage territorial et temporel comme éléments structurants, leur fondement répond également à un contexte commun : celui d'une tension ou d'une question à résoudre et d'un manque ou d'une absence d'informations suffisantes pour y répondre. Un problème de parité hommes/femmes, une dégradation environnementale, une crise de l'intermittence, de la violence dans les écoles, une inégalité d'accès aux soins... Les observatoires sont souvent nés dans des contextes à problèmes et apparaissent alors comme une solution : celle de disposer d'un dispositif neutre qui apporte des outils de représentation des situations, un lieu de référence où de la connaissance est produite à un niveau suffisamment en lien avec les préoccupations du terrain et suffisamment distancié pour ne pas être instrumentalisée.

Dans les représentations collectives, pour certains, ces dispositifs « méta » ne servent qu’à eux-mêmes. Pourtant force est de constater que dès qu'un problème est mis à la lumière, notamment dans les médias, les analyses produites par les observatoires sont utilisées, comme si ces derniers avaient anticipé sur les problématiques en jeu dans un secteur. De manière rigoureuse, un observatoire est un dispositif qui " agrège et enrichit des informations produites en interne par les organisations. Il les exploite, les analyse, diffuse et valorise les résultats. Il peut également les croiser avec d'autres sources nationales ou européennes. À aucun moment, cette démarche ne se substitue aux productions internes de chaque institution tels les bilans d'activités ou les rapports de gestion » (10). Ainsi l'observation est une modalité particulière de production de connaissances, reposant sur un processus itératif et permanent de production, de diffusion d'informations, d'analyses, validées par leurs conseils d'administration. Ce sont des espaces de synthèse, de capitalisation, d'analyse au service de l'ensemble des acteurs dans leur plus grande diversité. Les observatoires sont des dispositifs de l'« articulation », comme le souligne Jean Pierre Saez, directeur de l'Observatoire des Politiques culturelles (11), parce qu'ils visent à être une plateforme d'échange d'informations à destination des différents intéressés d'un domaine - les professionnels, les financeurs, les publics... Ils sont également à l'articulation avec le monde de la 
Recherche, en particulier Sciences humaines et sociales. Car si les deux mondes sont bien producteurs de connaissances, les finalités de leurs actions respectives se distinguent, voire même se complètent (12). Les observatoires se construisent à partir d'une question bien réelle et puisent dans l'avancement des recherches pour y trouver les outils, les modes d'analyse, les protocoles d'enquêtes. Ce positionnement de l'entre d'eux, implique que les équipes des observatoires ont tendance à être pluridisiplinaires, composées de statisticiens, démographes, experts du domaine, géographes, économistes, informaticiens...

Le rôle des observatoires est de produire des éléments qui aideront à la prise de décision ultérieure. Donner l'information, la rendre publique, participer à des débats publics relèvent de leurs activités principales. Ils sont ainsi souvent rattachés à un centre de ressources ou une activité de formation ou de publication.

\section{Évaluation ef observation : quelles articulations?}

Comme le soulignent Joëlle le Marec et Serge Chaumier dans l'article précédent, évaluation et observation sont des termes «valises » dont les multiples usages contribuent à une grande confusion. Cette absence de rigueur induit également une projection des propres fantasmes et peurs de chacun, quand on parle de dispositif d'observation ou de protocole d'évaluation. Une modeste clarification nous paraît ici nécessaire, pour mieux en souligner la différence et l'articulation.

L'observation n'est pas l'évaluation et vice versa, mais il est vrai que les pratiques d'évaluation nourrissent les programmes d'observation. L'observation et l'évaluation ont en commun d'être des démarches d'analyse et de connaissance. Mais la première consiste à produire des connaissances soit en mobilisant une information déjà disponible au sein d'autres organismes, soit en produisant des données spécifiques non disponibles par ailleurs alors que la seconde interroge l'efficience et l'efficacité dans la réalisation d'objectifs implicites et explicites d'une action ${ }^{(13)}$. Ainsi l'observation vise-t-elle à produire un état des lieux sur son objet d'étude, alors que l'évaluation travaille sur les valeurs d'une action, à partir notamment d'une lecture des écarts entre les objectifs définis préalablement et leurs résultats ou leurs effets réels. Elle renvoie implicitement à la question de la norme et de la normalité. La première relève d'une approche macro sur un secteur, un territoire, une tendance ; l'échelle de la seconde est celle du projet ou d'une action.
Poursuivant la même logique, les temporalités de ces deux démarches sont également différentes. Pour saisir l'évolution, l'observation ne peut être qu'une activité pérenne, qui se nourrit et prend sens dans le temps. La durée d'une évaluation recouvre celle du projet ou d'une politique à évaluer avec son éventuelle reconduction ou renouvellement. Mais c'est surtout dans leurs finalités que les divergences sont importantes : un des principes forts de l'observation est d'être transparent, de mettre à disposition et de rendre publique l'ensemble des données; les résultats de l'évaluation appartiennent aux commanditaires de l'étude et restent souvent confidentiels, la décision de les rendre publique repose sur leur souhait.

Dans un contexte de surveillance des dépenses publiques, le terme évaluation est souvent associé à une méthode contribuant à la rénovation du service public et à la modernisation de l'État. Dès 1990, l'évaluation des politiques publiques donne lieu à un décret qui stipule qu'«évaluer les politiques publiques consiste à mesurer les effets qu'elles engendrent et à chercher si les moyens juridiques, administratifs et financiers mis en œuvre produisent les effets qu'on en attend» (14). Les trois éléments constitutifs de l'évaluation sont dans une logique de décision publique: les objectifs, les moyens et les résultats ${ }^{(15)}$. Tout l'enjeu est de vérifier l'équilibre de ce triangle portant une politique. L'analyse du rapport objectifs-moyens interroge la question de la pertinence du projet, notamment à vérifier que les moyens sont adéquats aux objectifs énoncés. Le second rapport, celui des objectifs et des résultats vérifie que les résultats attendus ont été atteints. Et enfin le troisième, celui du rapport moyens-résultats relève de l'efficience : il vise à vérifier si les moyens mis en œuvre sont pertinents pour les résultats visés.

L'observation n'est pas étrangère à l'évaluation des politiques publiques, mais si l'évaluation a tendance à catégoriser les choses, « objectifs accomplis ou objectifs non accomplis», l'observation se veut être un outil de co-construction et de dialogue. Car nombreux sont les observatoires qui se positionnent non pas comme des dispositifs d'expert - donner des informations, produire de la connaissance et faire des propositions, des suggestions - mais plutôt comme des dispositifs favorisant la participation, voire même la coopération (voir l'exemple de la Fédurok décrit par Fabien Lacaille) : ils recueillent les données brutes et ne présentent pas d'interprétations, incitent les acteurs à interpréter euxmêmes leurs données, prennent en compte leur expertise et réflexivité et recherchent la pluralité des regards. Autant la première logique relève d'une 


\section{Le coopérahif mis en osuvre: I'exemple de la Fédurok}

\section{Un dispositif d'observation adapté}

Parmi les nombreux dispositifs d'observation existants, certains réseaux développent un mode participatif. Un des plus élaborés dans le milieu culturel est sans doute l'exemple de la Fédurok (Fédération de lieux de musiques amplifiées/actuelles) qui a développé depuis 1999 un dispositif d'observation participative et partagée (www.la-fedurok.org)

Face au manque de données concernant leur domaine d'activités, dû notamment à l'absence d'organisme en mesure de fournir ces informations, la Fédurok a en effet décidé de mettre en place en son sein un dispositif à même de les recueillir. Il s'agissait pour cette fédération de mieux se connaître ou plutôt de mieux connaître la structuration, les besoins de ses adhérents mais également de disposer de données à même de présenter la réalité économique et sociale aux décideurs et partenaires.

Dès le départ, le souhait d'impliquer les adhérents à tous les niveaux de l'observation (élaboration du questionnaire, alimentation en ligne, analyse des résultats) s'est affirmé. Cette implication permettait d'avoir une grille qui corresponde au plus près des réalités du terrain ; elle permettait aussi d'éviter le refus de répondre et la méfiance par rapport aux données chiffrées.

Ce dispositif d'observation s'est mis en place en plusieurs phases : - élaboration d'une grille de 1200 questions couvrant les quatre grands champs que sont le lieu, le projet, l'équipe et le territoire de la structure consultée ;

- développement de cette grille d'observation en base de données informatiques;

- à la mise en ligne de cette base de données informatique sur le site Internet de la Fédurok ;

- alimentation directe de la base de données en ligne par les membres du réseau de la Fédurok ;

- visite in situ (de 1 à 3 jours) de chacun des lieux adhérents avec rencontres et entretiens individuels pour les membre des équipes permanentes ;

- mise en place de requêtes et restitutions statistiques en temps réel sur le site Internet de la Fédurok à partir de la base de données ;

- production d'analyses et de synthèses.

Mettre en place un tel dispositif demande du temps et celui de la Fédurok, rejoint depuis par la FSJ (Fédération des scènes de Jazz et de Musiques Improvisées) est pleinement opérationnel depuis 2006. Si l'outil informatique a récemment évolué en 2009, la Fédurok reste fidèle à la philosophie participative de son dispositif et permet, chaque année, à ses membres (72 en 2009) de disposer d'un tableau de bord de leur activité et d'une analyse des résultats.

\section{Que faut-il retenir de cette expérience?}

Si tous les aspects ne sont pas directement applicables à d'autres champs culturels et notamment concernant la visite in situ de tous les membres du réseau, quelques points clefs sont cependant à retenir.

\section{Un objectif commun}

Les participants à un dispositif d'observation, qu'il soit imposé ou volontairement adopté, constituent de fait un réseau. Dans la mesure où la participation est volontaire, cela sous-entend que l'on se reconnaît un objectif commun, partagé avec les autres membres du dispositif. Ce partage d'un objectif commun est à la base de la réussite d'un tel dispositif. La première question à se poser est donc « quel(s) objectif(s) partageons-nous ? ».

\section{Une réelle dimension participative}

Beaucoup réduisent d'ailleurs la notion de «participatif » à une alimentation directe de la base de donnée par une application en ligne du questionnaire. Même si un dispositif participatif ne peut se résumer à cela, soulignons l'avantage que cela procure en évitant le long travail de traitement pour rentrer les données dans la base avant même de consacrer du temps à l'analyse même des résultats. Moins de temps pour le traitement, c'est plus de temps pour l'analyse. Trop d'acteurs, de responsables sur le terrain pointent l'opacité dans laquelle sont réalisés les questionnaires sans consultation aucune. À cela s'ajoute le manque de retour sur les résultats et surtout leur utilisation. Le « participatif » peut donc commencer dès l'élaboration de la grille d'enquête afin d'intégrer les remarques des acteurs de terrain sur la pertinence de certains points, de certains indicateurs. Et la participation peut continuer en faisant évoluer la grille, en la complétant. Les utilisateurs étant les plus à même de remarquer l'adéquation des critères avec les problématiques du terrain. Le moment de la restitution constitue également un point important pour permettre de ressortir des recommandations.

\section{Un effort de dynamisation}

Mais encore faut-il que ce réseau constitué continue de vivre audelà de sa mise en place. Celle-ci nécessite un effort important mais l'effort humain nécessaire à sa « survie » ne doit pas être négligé. Un travail de dynamisation du réseau doit sans cesse être mené. Sur un aspect pratique, cela consiste à relancer régulièrement les acteurs du réseau pour qu'ils continuent d'alimenter en informations le dispositif. Surtout, il est essentiel que le dispositif continue de produire un intérêt pour les participants, que ce soit par la richesse de sa base de données ou par les analyses qui en découlent. À l'échelle de la Fédurok, cela a nécessité le recrutement d'un sociologue entièrement dévolu à ces missions.

FABIEn LACAiLle Chargé d'étude Observatoire du Patrimoine et de la Culture Scientifiques et Techniques 
logique d'expertise autant la seconde est avant tout une dynamique où l'observatoire est un animateur du milieu : l'observatoire s'arrête là où un choix de société s'impose, l'observatoire s'efface au profit de ceux qui nourrissent leur projet. Car l'objectif n'est pas de qualifier seulement les choses mais de proposer une dynamique de changement en réponse au problème qui constitue l'objet même de l'observation.

\section{Un Observatoire pour le Patrimoine et la Culture Scientifiques et Techniques}

Si la pratique de l'évaluation muséale a été amplifiée par les musées des sciences, notamment par des approches en didactique puis sciences de l'information et de la communication, celle de l'observation a été menée par quelques projets sans aucun lien apparent, avec des objectifs, délibérés ou pas, de mieux saisir le monde de la muséographie et de la médiation des sciences. Quelques exemples qui ne rendent pas compte de la liste des projets de manière exhaustive.

Les plus connus sont les dispositifs de type Observatoires des publics appliqués à des structures qui, soit disposent des moyens humains ou financiers (16), soit participent à des programmes d'étude appliqués à l'échelle de plusieurs institutions (17). Notons qu'il existe également différents projets d'inventaires des acteurs de la Culture Scientifique et Technique (CST) ${ }^{(18)}$ et du patrimoine scientifique et technique qui assurent les fonctions essentielles de recensement, de capitalisation et de mise en visibilité ${ }^{(19)}$. Ces projets sont soit portés par des fédérations d'acteurs ou les têtes de réseaux, d'ambition nationale ou locale, soit rentrent dans le cadre des grands programmes de recensement du patrimoine à caractère scientifique. Des informations et données existent également, de ci de là, sur des sujets tels que l'emploi, les pratiques culturelles... en lien avec le champ de la CST, dans des grandes enquêtes nationales ou européennes. Dans le champ

\section{L'observation du champ professionnel de la culture scientifique en Europe}

Qu'en est-il de l'observation du patrimoine et de la culture scientifique en Europe ? Voici un premier panorama de dispositifs d'observation européens existants qui répondent à des missions d'observation, d'analyse, de partage (diffusion et dialogue) et de prospective de ce champ spécifique : une première liste car leur émergence se confirme - des structures qui gèrent à destination des professionnels, des banques de données publiques, la plupart du temps associées à un portail Internet, et qui produisent de manière épisodique des synthèses, des analyses sur ces données.

\section{Observatoires à vocation nationale}

- Science center netzwerk Austria (Autriche) : www.science-center-net.at/

Mapping Science Center Activities in Austria

- Institut für Museumsforschung (Allemagne) : www.smb.spk-berlin.de/ifm/

- Department of Buisiness, Industry \& Skills (Royaume- Uni) :

www.dius.gov.uk/science/science_and_society/mapping_the_landscape

Mapping the lanscape project

- Association for Science and Discovery Centres

(Royaume-Uni) : http://sciencecentres.org.uk/

Impact surveys

En devenir

- Observatoire du Patrimoine et de la Culture Scientifiques et Techniques (France) : www.ocim.fr/

\section{Observatoires à vocation supranationale}

- Observatori de la Comunicació Científica (International) : www.upf.edu/occ/

- Observa-Science in society (International) : www.observa.it/

- Ecsite (Europe) : www.ecsite.eu/?p=6385

- Observatoire Science, Politique et Société, université de Lausanne (Suisse) : www.unil.ch

En devenir

- EU/DG Recherche-PCRD 7 (Europe) : http://cordis.europa.eu/

Monitoring research and policy activities of science in society (MASIS) service contract

\section{Banques de données publiques associées} à des portails Internet

- Ciência Viva (Portugal) : www.cienciaviva.pt/

Data base of projects and forum

- Observatori Científic de la Ciutat MediterràneaOCCM (Barcelone, Espagne) : www.bcn.es/medciencies/

Medsciences portal

Jean-Louis Lacroix

Chargé de projet Observatoire et Europe 
de la culture, ces informations sont publiés dans les travaux du Département des Études, de la Prospective et des Statistiques du ministère de la Culture et de la Communication. Dans la problématique SciencesSociété, problématique émergente notamment dans les programmes de recherches nationaux ou de la commission, la perception et la représentation de la science et du monde de la recherche par nos concitoyens sont analysées dans les grandes enquêtes de type Eurobaromètres. Des études approfondies sont également initiées par la fédération de certaines familles de la CST, notamment les familles de l'Éducation populaire ${ }^{(20)}$, les Parcs naturels régionaux ${ }^{(21)}$... Le champ du patrimone et des institutions culturelles à caractère scientifique et technique se retrouve également ausculté par le monde du tourisme, notamment par le biais du baromètre de l'Agence de Développement touristique de la France qui recense entre autres l'ensemble des structures d'une fréquentation annuelle dépassant les 10000 visiteurs par an.

Ces quelques exemples témoignent que des informations et données de type qualitatives et quantitatives sur les structures, les acteurs et les publics existent, mais de manière éparpillée et avec une faible valorisation. Il apparaît bien utopique de regrouper toutes ces initiatives aussi riches les unes que les autres et qui ont été établies à chaque fois dans des logiques qui leur sont propres. Cette pluralité des protocoles méthodologiques annule toute grille de lecture commune, pourtant indispensable pour saisir une vision d'ensemble des actions publiques de la CST. Ce manque de données, notamment quantitatives et extrapolables, a été déclencheur dans l'idée de construire un dispositif d'observation centré sur les actions publiques de patrimoine et de médiation scientifiques. Mais la création d'un observatoire pour ce champ spécifique n'est pas seulement une réponse au souhait de disposer de chiffres et de tableaux de bord. Des éléments plus structurels à ce champ incitent à construire un outil qui garde en mémoire les initiatives passées et puisse repérer les enjeux de demain.

Une des raisons, et qui est récurrente dans la genèse des observatoires comme nous l'avons souligné plus haut, est que le champ du Patrimone et de la Culture Scientifiques et Techniques (PCST) est en pleine mutation, et que cette dynamique a des conséquences sur la visibilité de ce secteur à l'échelle des différents territoires. Sans prétendre à une analyse approfondie des mutations de ce secteur qui est l'objet de spécialistes, nous souhaitons mettre ici en lumière succinctement quelques éléments qui contribuent à un manque de visibilité et donc d'« impact » au sens large. Déjà la Culture Scientifique et Technique relève au niveau étatique, d'une grande variété de ministères, sans dynamique évidente de transversalité (22). La CST se retrouve au confluent d'univers comme celui de l'Éducation, de la Culture, de l'Enseignement supérieur, de la Recherche, du Tourisme, de la Communication... des domaines qui, notons-le, disposent de leur propre observatoire.

Bien plus la CST est dans une logique de décentralisation. Nombreuses sont les collectivités territoriales Conseils généraux, Conseils régionaux, Villes - qui ont investi dans des actions et projets parfois très ambitieux de CST car ils représentent pour eux un bon moyen de valorisation et de dynamisation de leurs territoires (23). Un autre point est celui de l'augmentation et la diversification des acteurs qui participent à des activités de médiation scientifique : les entreprises, les universités, les collectivités locales, les sociétés privées d'industrie culturelle... Notons que de nouveaux acteurs (pôles de compétitivité, universités, société civile...) ont pris place à côté des acteurs plus traditionnels (musées, muséums, centres de science...), sans parler des univers comme les média qui sont toujours restés à distance tout étant une des premières sources d'information pour le public. Un premier travail de représentation de ces producteurs et d'acteurs de la CST présenté ci-joint montre que la famille est composée de sous-familles qui sont parfois dans leur logique propre et qui ne connaissent pas leur «voisins » et ne pensent pas à des logiques de partenariat. Ainsi le public dispose-t-il de discours sur les sciences qui ne sont pas pensés en complémentarité ou qui parfois font doublon ou se parasitent. Aussi notons des tendances au sein d'une même famille où des sousfamilles se cristallisent autour de nouvelles dynamiques s'éloignant des modèles de références, fragilisant ainsi la fédération de ces acteurs (24).

Une première phase d'instruction pour le montage de cet observatoire a permis l'expression des acteurs sur leur besoin de se repérer dans ce paysage mouvant, à l'heure de la mondialisation et de la montée de la territorialisation (25). Si la question des chiffres et des données a provoqué des craintes dans ce contexte d'une évaluation très présente, le besoin d'un lieu d'échange et de distanciation et de compréhension de l'impact et par là même du sens de leurs activités s'est largement exprimé. 
Inventorier, analyser et accompagner : les grandes lignes de l'Observatoire de I'OCIM

En 2007, le ministère de l'Enseignement supérieur et de la Recherche a charger l'OCIM d'instruire lidée d'un projet d'Observatoire du Patrimoine et de la Culture Scientifiques et Techniques. Plusieurs phases se sont succédées : la première a consisté en une consultation individualisée d'une série des acteurs de la CST sur leur besoin et attente en termes d'outils d'observation (26), la seconde a travaillé sur l'idée de mise en œuvre d'un dispositif participatif et coopératif (27) et enfin la troisième a établi un cadrage général d'organisation des différents pré-chantiers avant son lancement officiel ${ }^{(28)}$. De ces trois phases de travail, complétées par un travail de benchmarking sur les autres observatoires, émerge le projet de l'Observatoire de l'OCIM que nous présentons de manière synthétique, son positionnement et sa définition pouvant être amenés à évoluer, comme ce fut le cas pour de nombreux autres observatoires.
L'Observatoire de l'OCIM aura pour mission de rendre visible les actions publiques du patrimoine et de culture scientifiques et techniques, et de produire des informations et des connaissances qui nourriront l'élaboration des politiques de PCST dans un contexte de décentralisation et de modification de rapport aux savoirs. L'observatoire se veut un outil d'objectivation des réalités et de dialogue.

Son champ d'observation se positionne autour de deux cercles : le premier qui fera l'objet d'une analyse plus complète et qui est le périmètre naturel de l'OCIM, à savoir les lieux du patrimoine et de la muséographie des sciences et enfin le second, qui pourra faire l'objet d'analyses à la marge en vue d'observer les évolutions et tendances globales, à savoir les actions publiques de médiation scientifique.

Ses missions se déclinent à ce jour selon quatre axes : la production et la diffusion de données quantitatives et qualitatives et d'analyses sectorielles, territoriales et thématiques, en priorité sur le premier cercle ; un outil de veille et de prospective sur les domaines qui peuvent influer les politiques de PCST ;

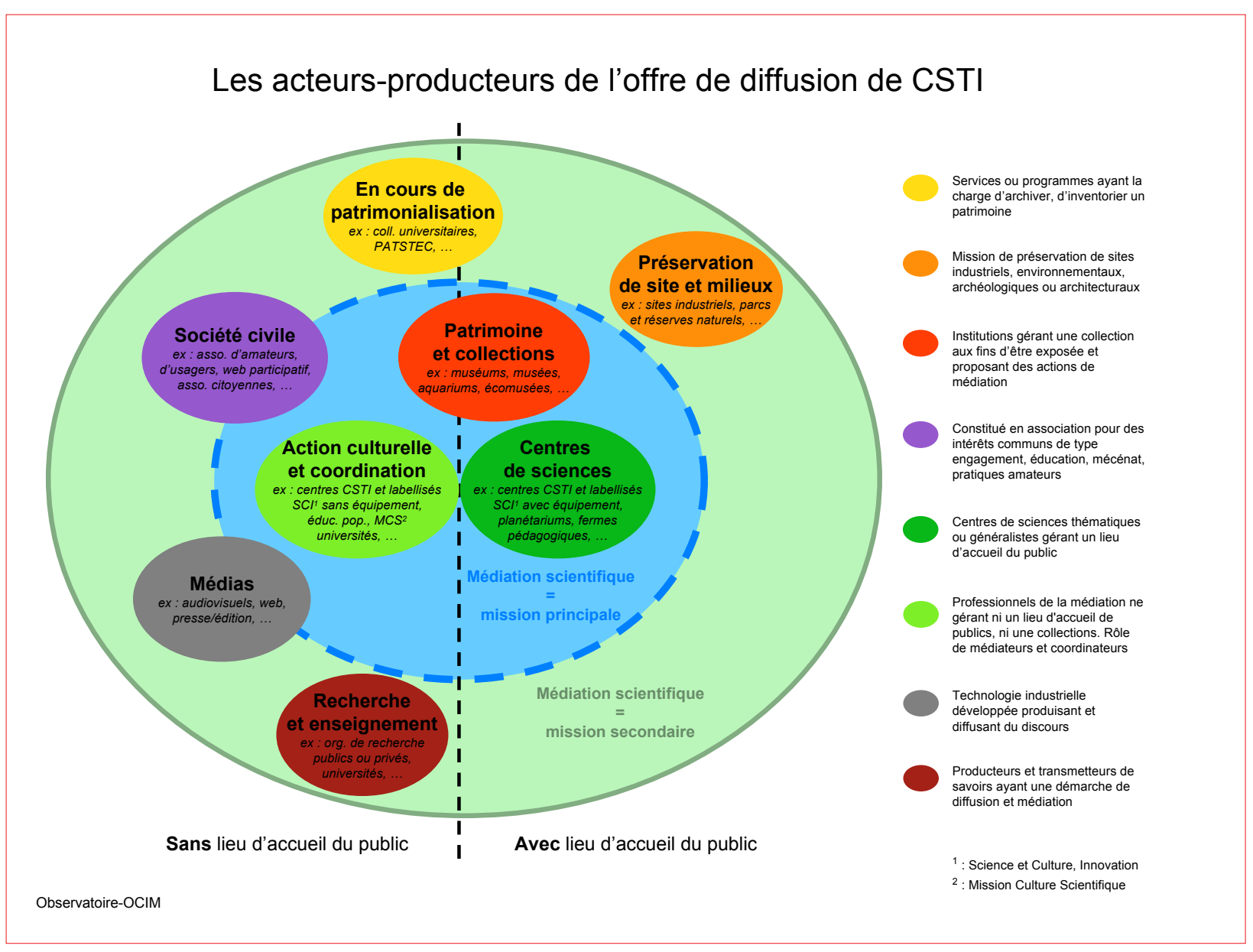


une plateforme de rencontre et dialogue entre les différents acteurs impliqués dans des programmes de PCST ; et une assistance technique et méthodologique en termes d'évaluation et de dynamique partenariale. L'ensemble de ces activités s'articule autour de trois mots clefs : inventorier, analyser et accompagner. Le volet Inventorier consistera à établir une base de données actualisée décrivant l'ensemble des lieux de muséographie et de patrimoine scientifique (collections, activités, publics emploi...). Ce dispositif, qui est en cours de conception, vise à être un dispositif coopératif et participatif, l'idée étant d'impliquer les acteurs tout au long du process.

Le volet Analyser portera sur des sujets spécifiques comme l'économie, l'emploi et la formation, les financements publics et les politiques des territoires, les publics et les pratiques culturelles. Les méthodes seront ad hoc, ancrées dans différentes disciplines du monde des Sciences humaines et sociales. Un travail de veille consistera à repérer les évolutions dans l'offre de médiation scientifique et de suivre également les méthodologies d'enquête.

Enfin, concernant le troisième volet, celui d'Accompagner, rappelons que comme de nombreux observatoires cités plus haut, l'Observatoire de l'OCIM a l'intention de travailler à la charnière des services de l'État, des collectivités territoriales, des professionnels de la médiation scientifique, avec ou sans base patrimoniale, et de jouer également le rôle d'interface entre le monde de la recherche en sciences humaines et sociales - ses questionnements, ses avancées théoriques - et le monde des professionnels de la médiation scientifique. Ainsi proposera-t-il des rencontres sur des thématiques qui auront fait l'objet de travaux par l'observatoire au préalable, l'idée étant de favoriser le dialogue et l'échange entre les différents acteurs de ce champ.

\section{De l'observation à la reconnaissance d'un secteur culturel}

Le champ de la Culture Scientifique et Technique apparaît être un des derniers à ne pas encore avoir pu disposer d'un tel outil structurant. Ce qui pourrait expliquer les multiples attentes exprimées par de nombreux interlocuteurs. Mais comme le rappelle René Rizzardo, fondateur de l'Observatoire des Politiques Culturelles: « un observatoire modifie le champ qu'il observe ». Bien plus, comme l'a montré l'analyse de l'usage des observatoires, leurs créations vont de pair avec la professionnalisation du champ qu'ils observent (29). Espérons que l'Observatoire du Patrimoine et de la Culture Scientifiques et Techniques porté par l'OCIM, en collaboration avec les autres observatoires évoqués plus haut, participera à une meilleure connaissance et par là même reconnaissance de la CST en tant que secteur culturel à part entière. L'Observatoire de l'OCIM souhaite développer une démarche rigoureuse, durable et dont la neutralité devra être reconnue pour qu'il devienne un outil utile pour tous, les décideurs, les acteurs et les publics.

Le lancement officiel de ce dispositif donne lieu à une consultation nationale qui prend forme de Rencontres les 26 et 27 novembre à Dijon, rencontres conçues pour être riches en interventions et ateliers de discussion. Ces rencontres illustrent l'esprit de ce futur Observatoire, esprit que l'on retrouve dans le « $\mathrm{C} »$ de l'OCIM, - Coopération -, l'Observatoire du Patrimoine et de la Culture Scientifiques et Techniques s'inscrit dans une logique de co-construction, non seulement avec les autres dispositifs d'observation qui contribuent à une connaissance spécifique ou à la marge de ce champ mais également avec les acteurs qui souhaitent s'investir dans ce dispositif. Co-construction pour, espérons-le, une co-écriture de l'évolution du champ de la culture scientifique et technique.

\section{Notes}

(1) Nous renvoyons à ce site qui inventorie une liste impressionnante d'observatoires, www.adminet.com/obs

(2) Exemple : Observatoire des territoires créé par décret et placé auprès du ministre chargé de l’Aménagement du Territoire

(3) Exemple : Observatoires régionaux de l'Environnement

(4) Exemple : Observatoire Science, Politique et Société est rattaché à la faculté des Sciences sociales et politiques de l'université de Lausanne

(5) Exemple: Observatoire des Sciences et des Techniques

(6) Exemple : dispositif d'observation de la Fédurok

(7) Exemple : Observatoire régional du Spectacle vivant de l'Agence régionale du Spectacle vivant, Poitou Charente

(8) Exemple : Observatoire citoyen des choix scientifiques de l'association Fondation Sciences Citoyennes

(9) Exemple : Observatoire des Publics, département Évaluation et Prospective de la Cité des Sciences et de l'Industrie

(10) Observer la culture en région. Contribution du groupe de travail sur l'observation en région, Édition Oppes, 2003, p. 15.

(11) L'observatoire des politiques culturelles, vingt ans d'accompagnement de la vie culturelle, Policultures, la lettre des politiques culturelles et artistiques, $\mathrm{n}^{\circ} 136$, mai 2009, pp. 2-3. Si le Département Études, Prospective et Statistiques du ministère de la Culture et de la Communication peut être considéré comme un pôle ressource producteur d'études aux moyens conséquents, comme les enquêtes successives Les 
pratiques culturelles des français, l'Observatoire des Politiques Culturelles de Grenoble (qui émane du DEPS) s'affiche clairement être un dispositif porteur d'une dynamique à l'articulation du monde de la culture, du monde politique et du monde de la recherche. Si le premier peut être perçu comme le lieu de référence qui produit, organise et capitalise des informations, le second est plus proche des acteurs en organisant des formations et des séminaires de réflexion.

(12) Les observatoires et les centres de recherche cherchent à produire tous les deux de la connaissance, mais si les premiers se doivent d'être en résonance avec le terrain, les seconds demeurent dans un fonctionnement universitaire : système d'évaluation propre au monde universitaire, création de problème théoriques, jargon spécifique...

(13) Observer la culture en région, opus cite, p. 19

(14) L'évaluation au service des politiques culturelles locales. Élements pour la réflexion et l'action. La Documentation française, 1994, p. 7.

(15) Idem, pp. 22-23.

(16) Comme la Cité des Sciences et de l'Industrie, le Museum national d'Histoire naturelle ou certains CCSTI comme l'Espace des Sciences ou La Casemate.

(17) Comme l'ensemble des musées qui ont participé aux enquêtes de publics initiés par l'Observatoire permanent des Publics de la Direction des Musées de France.

(18) Comme par exemple les inventaires réalisés par les CCSTI considérés comme les têtes de réseau d'un territoire.

(19) Comme le programme PATSTEC.

(20) Voir les travaux du CIRASTI ou de l'AFA.
(21) Voir les études de la Fédération des Parcs naturels régionaux de France.

(22) Ministère de l'Enseignement supérieur et de la Recherche, ministère de la Culture et de la Communication, ministère de l'Écologie, de l'Énergie, du Développement durable et de la Mer, ministère de la Santé et des Sports...

(23) Voir Politique (une) culturelle à la rencontre d'un territoire. Culture scientifique, technique et industrielle en région Lorraine 1980-1995, par Marie-Jeanne Choffel-Mailfert, Éditions l'Harmattan, 2000.

(24) Une recherche portant sur l'évolution des planétariums et de leur réseau de référence. Voir Belaën, F. Les planétariums : observatoire d’une tendance, Culture \& Musée $\mathrm{n}^{\circ}$ 10, 2007.

(25) Gachet, L.-J., Chevolet, A. et Breton, M. Rapport Comité d'Orientation et de Pilotage, OCIM, juillet 2008.

(26) Idem

(27) Gachet, L.-J. et Lacaille, F. Rapport Comité d'Orientation et de Pilotage, OCIM, octobre 2008.

(28) Gachet, L.-J., Belaën, F. et Lacaille, F. Rapport Comité d'Orientation et de Pilotage, OCIM, février 2009

(29) Extrait du Rapport annuel 2002 de l'Inspection générale des Affaires sociales Politiques sociales de l'État et territoires à propos de l'Observatoire régional de la Santé : «après une décennie, marquée par une certaine fragilité et une incontestable précarité des ORS, la décennie suivante est marquée par la professionnalisation de la santé publique et des ORS, les deux phénomènes se conjuguent naturellement». 